\title{
Iterative Fuzzy Support Vector Machine Classification
}

\author{
Alistair Shilton and Daniel T. H. Lai
}

\begin{abstract}
Fuzzy support vector machine (FSVM) classifiers are a class of nonlinear binary classifiers which extend Vapnik's support vector machine (SVM) formulation. In the absence of additional information, fuzzy membership values are usually selected based on the distribution of training vectors, where a number of assumptions are made about the underlying shape of this distribution. In this paper we present an alternative method of generating membership values which we call iterative FSVM (I-FSVM). Our method generates membership values iteratively based on the positions of training vectors relative to the SVM decision surface itself. We show that our algorithm is capable of generating results equivalent to an SVM with a modified (non distance based) penalty (risk) function. Experiments have been carried out on three real world binary classification problems taken from the UCI repository, namely the spambase dataset and the adult (census) dataset.
\end{abstract}

\section{INTRODUCTION}

Support vector machines (SVMs) are a class of non-linear binary classification algorithm based on the theory of structural risk minimisation (SRM) due originally to Vapnik [1], [2]. They are able to solve highly complex classification tasks [3], [4], [5], [6] without suffering from the problems of over-fitting that affect many other classification algorithms. Computationally speaking, the SVM training problem is a convex quadratic programming problem, meaning that local minima are not a problem.

SVMs work by implicitly (using the kernel trick) mapping all training data from input space into a (usually) higher dimensional feature space. An oriented linear hyperplane (decision surface) is constructed in this feature space such that it bisects the two classes of training vectors and maximises the perpendicular distance between itself and those points lying closest to it (the support vectors). If the classes are non-separable in feature space then the condition of strict separability is relaxed by adding a linear penalty (risk) term to the primal cost function to penalise any misclassifications.

Fuzzy support vector machines (FSVMs) [7] work similarly, except that a membership value is associated with each training vector (this membership value is incorporated into the penalty term to provide variable weighting). In this way the contribution of a training vector to the form of the decision surface may be moderated based on its relevance in comparison to the rest of the training set. Unless apriori information about the relevance of the training vectors (eg. vector age in time-varying systems) is available, the membership values are calculated based on the distribution of training vectors, with outliers being given proportionally smaller membership values than other training vectors.

Alistair Shilton and Daniel T.H. Lai are with the Department of Electrical and Electronic Engineering, The University of Melbourne, Melbourne, Australia (email: apsh@ee.unimelb.edu.au and d.lai@ee.unimelb.edu.au, respectively).
In this paper we introduce a new algorithm for the calculation of the membership values using an iterative FSVM (I-FSVM) that makes no a-priori assumptions about the shape of the distribution of the training vectors. Our method makes use of the result of the SVM training process and information about incorrectly classified training vectors (error vectors) to tune the membership values. The FSVM is then re-trained with these new values, and the process repeated either for a fixed number of iterations or until the membership values converge.

We show that, when it is allowed to run to termination, our I-FSVM method is theoretically equivalent to the standard SVM with a modified penalty term. We then provide some experimental results obtained using three datasets from the UCI repository [8], namely the adult (census) dataset, the spambase dataset and the abalone dataset. In all cases the I-FSVM algorithm is shown to outperform both the standard SVM and the FSVM method of [7].

The remainder of the paper is organised as follows. In section II we introduce the theory of the standard (non-fuzzy) SVM classification and FSVM classification [7]. Then, in section III we give a brief review of some standard methods for generating membership values, before introducing our iterative approach to this problem in section IV. Finally, in section $\mathrm{V}$ we present some practical results, followed by some discussion and conclusions in section VI.

\section{A. Notation and Terminology}

Throughout this paper column vectors will be written in lower case bold (eg. a, $\boldsymbol{\alpha}$ ) and matrices in upper case bold (eg. G). Transposition will be indicated by a superscript $T$ $\left(\mathrm{eg}, \mathbf{c}^{T}\right)$, and 1 will be used to represent a column vector of ones. We use $\mathbb{Z}_{N}$ to represent the integers modulo $N$ (so $\mathbb{Z}_{N}=\{0,1, \ldots, N-1\}$ ), $\mathbb{Z}^{+}$to represent the positive integers and $\mathbb{R}^{+}$to represent the positive reals.

We use the terms input space and feature space in the standard SVM sense. That is, input space is taken to mean the space of all training vectors $\mathbf{x}_{i}$, which we assume to be $\mathbb{R}^{d_{L}}$ where $d_{L} \in \mathbb{Z}^{+}$. Feature space is defined to be the space $\mathbb{R}^{d_{H}}$ where $d_{H} \in \mathbb{Z}^{+} \cup\{\infty\}$ in which the SVM implicitly constructs the decision surface, as discussed in the next section.

\section{Support Vector Machine Basics}

We begin by revising the theory of support vector machines for binary classification. The aim of binary classification may be stated as follows. Given a training set:

$$
\begin{aligned}
\Theta & =\left\{\left(\mathbf{x}_{0}, d_{0}\right),\left(\mathbf{x}_{1}, d_{1}\right), \ldots,\left(\mathbf{x}_{N-1}, d_{N-1}\right)\right\} \\
\mathbf{x}_{i} & \in \mathbb{R}^{d_{L}} \text { is the } i^{\text {th }} \text { input vector } \\
d_{i} & \in\{-1,+1\} \text { is the classification of } \mathbf{x}_{i}
\end{aligned}
$$


construct a classifier $f: \mathbb{R}^{d_{L}} \rightarrow\{-1,+1\}$ that can classify arbitrary vectors $\mathbf{x} \in \mathbb{R}^{d_{L}}$ into one of the two classes, -1 and +1 , in a manner consistent with the training set $\Theta$.

The SVM approach to this problem is to search for classification functions of the form:

$$
f(\mathbf{x})=\operatorname{sgn}(g(\mathbf{x}))
$$

where:

$$
g(\mathbf{x})=\mathbf{w}^{T} \boldsymbol{\varphi}(\mathbf{x})+b
$$

and $\varphi: \mathbb{R}^{d_{L}} \rightarrow \mathbb{R}^{d_{H}}$ is an implicitly defined map from $d_{L}$-dimensional input space to $d_{H}$-dimensional (where often $d_{H} \gg d_{L}$ ) feature space, $\mathbf{w} \in \mathbb{R}^{d_{H}}$ is the weight vector and $b \in \mathbb{R}$ is the bias.

Geometrically, then, the SVM approach involves the construction of a linear decision surface (directed hyperplane), defined by $\mathbf{w}$ and $b$, in $d_{H}$-dimensional feature space. Ideally this decision surface should bisect the positive and negative training vectors in feature space and thereby correctly classify the training set, so:

$$
d_{i} g\left(\mathbf{x}_{i}\right)=d_{i}\left(\mathbf{w}^{T} \boldsymbol{\varphi}\left(\mathbf{x}_{i}\right)+b\right)>0 \forall i \in \mathbb{Z}_{N}
$$

A training set for which this is possible is called separable. Assuming that the training set is separable, there will be an infinite number of possible separating hyperplanes satisfying (1), and hence an infinite number of possible choices for $\mathbf{w}$ and $b$. To select the best decision surface, the support vector machine attempts to maximise the margin of separation, which is defined to be the perpendicular distance between the decision surface and those images of training vector lying closest to it in feature space (which are known as support vectors). The optimal hyperplane may be found by solving [1]:

$$
\begin{aligned}
& \min _{\substack{\mathbf{w} \in \mathbb{R}^{d_{H}}, b \in \mathbb{R} \\
\text { such that: }}} L(\mathbf{w}, b)=\frac{1}{2} \mathbf{w}^{T} \mathbf{w} \\
& d_{i}\left(\mathbf{w}^{T} \boldsymbol{\varphi}\left(\mathbf{x}_{i}\right)+b\right) \geq 1 \quad \forall i \in \mathbb{Z}_{N}
\end{aligned}
$$

In most cases the training set will not be separable in feature space, and hence the optimisation problem (2) will be unsolvable. To overcome this difficulty the constraints in (2) are relaxed via the introduction of slack variables $\boldsymbol{\xi} \in \mathbb{R}^{N}$, and a penalty term (an measure of empirical risk) is added to the primal cost function $L(\mathbf{w}, b, \boldsymbol{\xi})$ to penalise any failure to meet the original, strict separability constraints. Using the usual linear penalty method the primal form of the SVM training problem is:

$$
\begin{array}{ll}
\min _{\mathbf{w} \in \mathbb{R}^{d_{H}}, b \in \mathbb{R}, \boldsymbol{\xi} \in \mathbb{R}^{N}} L(\mathbf{w}, b, \boldsymbol{\xi})=\frac{1}{2} \mathbf{w}^{T} \mathbf{w}+\frac{C}{N} \mathbf{1}^{T} \boldsymbol{\xi} \\
\text { such that: } & d_{i}\left(\mathbf{w}^{T} \boldsymbol{\varphi}\left(\mathbf{x}_{i}\right)+b\right) \geq 1-\xi_{i} \quad \forall i \in \mathbb{Z}_{N} \\
& \boldsymbol{\xi} \geq \mathbf{0}
\end{array}
$$

where the constant $C \in \mathbb{R}^{+}$controls the trade-off between empirical risk minimisation (and possible overfitting if $C$ is too large) and margin maximisation (and possible underfitting if $C$ is too small).

There are two main difficulties with the primal training problem (3). The constraint set is complicated (relatively

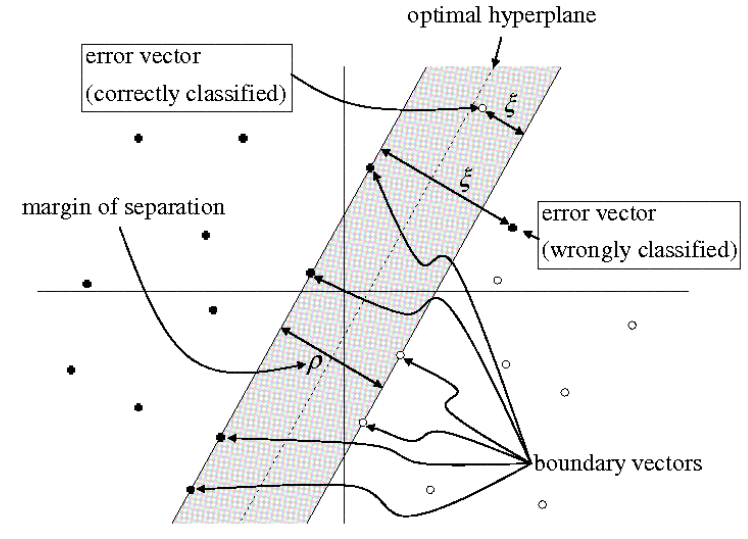

Fig. 1. Optimal hyperplane selection via max-margin, (inseparable case).

speaking), and the dimensionality of the problem is directly dependent on the dimension $d_{H}$ of feature space, which rather limits the acceptable range for $d_{H}$. Both of these problems may be overcome by re-expressing (3) in dual form, namely [1]:

$$
\begin{array}{ll}
\min _{\boldsymbol{\alpha} \in \mathbb{R}^{N}} Q(\boldsymbol{\alpha})=\frac{1}{2} \boldsymbol{\alpha}^{T} \mathbf{G} \boldsymbol{\alpha}-\mathbf{1}^{T} \boldsymbol{\alpha} \\
\text { such that: } & \mathbf{0} \leq \boldsymbol{\alpha} \leq \frac{C}{N} \mathbf{1} \\
& \mathbf{d}^{T} \boldsymbol{\alpha}=0
\end{array}
$$

where $\boldsymbol{\alpha} \in \mathbb{R}^{N}$ is the dual variable vector, $\mathbf{G} \in \mathbb{R}^{N \times N}$ is the hessian, $G_{i, j}=d_{i} d_{j} K\left(\mathbf{x}_{i}, \mathbf{x}_{j}\right) \forall i, j \in \mathbb{Z}_{N}$, and $K: \mathbb{R}^{d_{L}} \times \mathbb{R}^{d_{L}} \rightarrow \mathbb{R}$ is the kernel function, $K(\mathbf{x}, \mathbf{y})=$ $\varphi^{T}(\mathrm{x}) \boldsymbol{\varphi}(\mathrm{y})$, which may be any kernel function satisfying Mercer's condition [9] (knowledge of the explicit form of the map $\varphi$ is not necessary).

The decision function $g: \mathbb{R}^{d_{L}} \rightarrow \mathbb{R}$ may be written:

$$
g(\mathbf{y})=\sum_{i \in \mathbb{Z}_{N}} \alpha_{i} K\left(\mathbf{x}_{i}, \mathbf{y}\right)+b
$$

where [1]:

$$
b=d_{j}-\sum_{i \in \mathbb{Z}_{N}} \alpha_{i} K\left(\mathbf{x}_{i}, \mathbf{x}_{j}\right) \quad \forall j: 0<\alpha_{j}<\frac{C}{N}
$$

Note that each dual variable $\alpha_{i}$ is associated with a training pair $\left(\mathbf{x}_{i}, d_{i}\right) \in \Theta$. Based on the value of $\alpha_{i}$ training pairs may be classified as follows [1], [10]:

- Non-support vectors: $\alpha_{i}=0$ (and hence $d_{i} g\left(\mathbf{x}_{i}\right) \geq 1$ ).

- Boundary vectors: $0<\alpha_{i}<\frac{C}{N}$ (hence $d_{i} g\left(\mathbf{x}_{i}\right)=1$ ).

- Margin vectors: $\alpha_{i}=\frac{C}{N}$ (and hence $0<d_{i} g\left(\mathbf{x}_{i}\right) \leq 1$ ).

- Error vectors: $\alpha_{i}=\frac{C}{N}$ (and hence $\left.d_{i} g\left(\mathbf{x}_{i}\right) \leq 0\right)$.

where boundary, margin and error vectors are all categorised as support vectors. The feature space interpretation of this is shown in figure 1. It should be noted that the support vectors typically make up only a small fraction of all training vectors, and also that the distance of a point $\mathbf{x}_{i}$ from the decision surface in feature space is directly proportional to $|g(\mathbf{x})|$ ([1], [10]).

For later reference, note that:

$$
\xi_{i}=\max \left\{0,1-d_{i} g\left(\mathbf{x}_{i}\right)\right\} \quad \forall i \in \mathbb{Z}_{N}
$$




\section{A. Fuzzy Support Vector Machines}

When dealing with standard SVMs, all training vectors are presumed to belong entirely to either class +1 or class -1 , and moreover are assumed to have equal weight or relevance. However in many applications this assumption may not hold, as some training vectors may have more importance than others, and sometimes we may not be entirely certain that the purported classification $d_{i}$ of a training vector $\mathbf{x}_{i}$ is actually correct.

Fuzzy theory deals with these issues by saying that a training vector $\mathbf{x}_{i}$ belongs, for example, $90 \%$ to class +1 and $10 \%$ to class -1 . This may be achieved by associating a fuzzy membership $0<s_{i} \leq 1$ with each training pair $\left(\mathbf{x}_{i}, d_{i}\right)$ [7]. The pair is then regarded as having a membership of $s_{i}$ to class $d_{i}$; and a membership $1-s_{i}$ to class $-d_{i}$ (although the latter is usually ignored in FSVM theory [7]). This may be incorporated into the SVM framework by modifying the primal training problem as follows [7]:

$$
\begin{array}{cl}
\min _{\mathbf{w} \in \mathbb{R}^{d_{H}}, b \in \mathbb{R}, \boldsymbol{\xi} \in \mathbb{R}^{N}} L(\mathbf{w}, b, \boldsymbol{\xi})=\frac{1}{2} \mathbf{w}^{T} \mathbf{w}+\frac{C}{N} \mathbf{s}^{T} \boldsymbol{\xi} \\
\text { such that: } \quad d_{i}\left(\mathbf{w}^{T} \boldsymbol{\varphi}\left(\mathbf{x}_{i}\right)+b\right) \geq 1-\xi_{i} \quad \forall i \in \mathbb{Z}_{N} \\
\quad \boldsymbol{\xi} \geq \mathbf{0}
\end{array}
$$

where the error penalty (or empirical risk) of each training pair $\left(\mathbf{x}_{i}, d_{i}\right)$ is weighted by its membership $s_{i}$ of class $d_{i}$, so that those training pairs with smaller $s_{i}$ values (ie. those training pairs that are less relevant or reliable) will have less influence on the decision surface than those with larger $s_{i}$ values.

The dual formulation of the fuzzy SVM (FSVM) training problem is [7]:

$$
\begin{array}{ll}
\min _{\boldsymbol{\alpha} \in \mathbb{R}^{N}} Q(\boldsymbol{\alpha})=\frac{1}{2} \boldsymbol{\alpha}^{T} \mathbf{G} \boldsymbol{\alpha}-\mathbf{1}^{T} \boldsymbol{\alpha} \\
\text { such that: } & \mathbf{0} \leq \boldsymbol{\alpha} \leq \frac{C}{N} \mathbf{s} \\
& \mathbf{d}^{T} \boldsymbol{\alpha}=0
\end{array}
$$

and the decision function is the same as that of the standard SVM, namely (5), and:

$$
\xi_{i}=\max \left\{0,1-d_{i} g\left(\mathbf{x}_{i}\right)\right\} \quad \forall i \in \mathbb{Z}_{N}
$$

\section{Generating Membership Values}

A number of methods for generating membership values $s_{i}$ may be found in the literature. If the training set is time dependent then the membership may be based on the age of the data, with older data being given less weight (ie. smaller membership value $s_{i}$ ) than newer data. Alternatively, if the training vectors from one class are known to be less reliable than those from the other (or we have more samples from one class than another) then we may use:

$$
s_{i}= \begin{cases}s_{+} & \text {if } d_{i}=+1 \\ s_{-} & \text {if } d_{i}=-1\end{cases}
$$

Another approach (which we will be using for comparative purposes here) is to generate the fuzzy memberships $s_{i}$ based on the distribution of the two classes of training vector from our training set using the distance of points from the mean of vectors of the same class for the training set [7]. Under this scheme:

$$
s_{i}= \begin{cases}1-\frac{v_{i+}}{r_{+}+\delta} & \text { if } d_{i}=+1 \\ 1-\frac{v_{i-}}{r_{-}+\delta} & \text { if } d_{i}=-1\end{cases}
$$

wherein:

- $v_{i \pm}$ is the distance of training vector $\mathbf{x}_{i}$ from the class mean $\mathbf{x}_{ \pm}$of all training vectors of class \pm 1 .

- $r_{ \pm}$is the radius of the smallest ball centered at the class mean $\mathbf{x}_{ \pm}$that contains all training vectors $\mathbf{x}_{i}$ of class \pm 1 . That is:

$$
r_{ \pm}=\max _{i \in \mathbb{Z}_{N}: d_{i}= \pm 1} v_{i \pm}
$$

- $\delta>0$ is a constant included to avoid the case $s_{i}=0$.

The distances $v_{i \pm}$ may be measured either in input space or feature space. In input space:

$$
v_{i \pm}=\left|\mathbf{x}_{i}-\mathbf{x}_{ \pm}\right|
$$

where:

$$
\mathbf{x}_{ \pm}=\frac{1}{N_{ \pm}} \sum_{i \in \mathbb{Z}_{N}: d_{i}= \pm 1} \mathbf{x}_{i}
$$

and $N_{+}$and $N_{-}$are the number of training vectors in class +1 and -1 , respectively.

Substituting $\mathbf{x}_{ \pm}$into (11), replacing $\mathbf{x}_{i}$ with $\varphi\left(\mathbf{x}_{i}\right)$ and expanding we get the feature space expression:

$$
v_{i \pm}=\sqrt{\begin{array}{l}
K\left(\mathbf{x}_{i}, \mathbf{x}_{i}\right)-\frac{2}{N_{ \pm}} \sum_{j: d_{j}= \pm 1} K\left(\mathbf{x}_{i}, \mathbf{x}_{j}\right) \ldots \\
\cdots+\frac{1}{N_{ \pm}^{2}} \sum_{j, k: d_{j}=d_{k}= \pm 1} K\left(\mathbf{x}_{j}, \mathbf{x}_{k}\right)
\end{array}}
$$

It is difficult to say which of these schemes will work better for any given problem. As an aside, it may be noted that these equations may be used to generate fuzzy memberships based on the distribution of points in a feature space defined by any Mercer kernel, without restricting ourselves to input space (which may be recovered by choosing $K(\mathbf{x}, \mathbf{y})=\mathbf{x}^{T} \mathbf{y}$ in (12)) or the feature space implicitly used by the FSVM itself. It remains unclear whether there is any advantage to be gained from using kernels that do not measure distance in input space or the feature space used by the SVM.

\section{AN ITERATIVE SVM APPROACH TO GENERATING Membership Values}

Lin and Wang's method [7] is based on the assumption that outliers in the set of training vectors are less reliable, and hence of less importance, than other training vectors. As outliers are detected based solely on their relative distance from their class mean, this method may be expected to produce good results if the distributions of training vectors $\mathbf{x}_{i}$ of each class are spherical with central means (in the space used to calculate the memberships). In general, however, this assumption may not hold, which motivates us to seek a more universally applicable method.

Consider the set of training vectors $\mathbf{x}_{i}$ belonging to a given class $(+1$ or -1$)$. Let us assume that this set is sufficiently 
large to be a representative sample of the underlying distribution of training vectors in the form of a cloud of points in feature space. We further assume that the membership $s_{i}$ of any given vector $\mathbf{x}_{i}$ of class $d_{i}$ is a function of the density of training points of that class in the vicinity of $\varphi\left(\mathbf{x}_{i}\right)$ in feature space (ie. the lower the density of points of class $d_{i}$ in the vicinity of $\varphi\left(\mathbf{x}_{i}\right)$, the lower the membership value $s_{i}$ of that point).

Now, any reasonable decision surface in feature space (such as that given by the SVM itself) should only pass through areas sparsely populated by points of either class, and moreover should correctly bisect the more densely populated regions of feature space. Hence those points lying closest to, or on the wrong side of, the decision surface (ie. the margin and error support vectors) are more likely to be outliers than points lying in more densely populated regions of feature space. Error (incorrectly classified) support vectors, in particular, may be quite reasonably expected to be outliers.

This motivates our proposed method. Let us suppose that we have constructed a non-fuzzy SVM decision surface for our training set $\Theta$. We then calculate memberships $s_{i}$ using the equation:

$$
s_{i}=h\left(\xi_{i}\right)
$$

where $h$ is some continuous non-increasing function satisfying:

$$
\begin{aligned}
& \lim _{\xi \rightarrow 0+} h(\xi)=1 \\
& 0<h(\xi) \leq 1 \quad \forall \xi \geq 0
\end{aligned}
$$

and $\xi_{i}$ is the slack error (6). Using these memberships a fuzzy SVM boundary may be constructed, and if desired the process may be iterated using this new (and presumably more accurate) decision boundary either a fixed number of times or until the membership vector $\mathbf{s}$ converges. We call our method iterative fuzzy SVM (I-FSVM).

To be specific, our method of generating membership values is as follows (noting that incremental training [11] may be used at step 2 to minimise the re-training time for each iteration):

1) Set $\mathbf{s}=1$.

2) Solve the FSVM dual training problem (8).

3) For all $i \in \mathbb{Z}_{N}$ set (where $0<\mu<1$ ):

$$
s_{i}=s_{i}^{\text {previous }}+\mu\left[h\left(\xi_{i}\right)-s_{i}^{\text {previous }}\right]
$$

4) Exit if termination condition met.

5) Otherwise repeat from step 2.

We consider two possible termination conditions. The first (and simpler) termination condition is to stop after a fixed number $(n)$ of iterations, which we will refer to as I-FSVM $n$. The second termination condition is to continue until the rate of change of $\mathbf{s}$ becomes sufficiently small, indicating that the class membership vector $\mathbf{s}$ has converged to some value. Specifically, the second termination condition we consider is:

$$
\left|s_{i}-s_{i}^{\text {previous }}\right| \leq 10^{-3} \quad \forall i \in \mathbb{Z}_{N}
$$

which we will refer to as I-FSVM $\infty$. While we have not proved that I-FSVM $\infty$ will terminate in finite time, it is our experience that it does so (i.e. we have yet to encounter any cases of non-convergence).

We consider two possible membership functions in this paper. The first of these is the function:

$$
h_{\mathrm{cnt}}(\xi)=h(\xi)= \begin{cases}1 & \text { if } \xi<1 \\ \frac{1}{\xi} & \text { otherwise }\end{cases}
$$

where the membership is inversely proportional to the distance from the hyperplane. The second is the function (motivated in the following section):

$$
h_{\text {sig }}(\xi)=\operatorname{sech}^{2}(\xi)
$$

\section{A. Generalized Cost and the I-FSVM $\infty$}

We now consider I-FSVM $\infty$ in more detail. To begin, note that upon termination $s_{i}=h\left(\xi_{i}^{*}\right)$ for all $i$ (to within some predefined tolerance), where $\xi^{*}$ is the optimal slack variable vector for the fuzzy primal training problem (7).

Consider the generalised primal SVM training problem [12]:

$$
\begin{array}{ll}
\min L(\mathbf{w}, b, \boldsymbol{\xi})=\frac{1}{2} \mathbf{w}^{T} \mathbf{w}+\frac{C}{N} \sum_{i \in \mathbb{Z}_{N}} l\left(\xi_{i}\right) \\
\text { such that: } \quad d_{i}\left(\mathbf{w}^{T} \boldsymbol{\varphi}\left(\mathbf{x}_{i}\right)+b\right) \geq 1-\xi_{i} \quad \forall i \in \mathbb{Z}_{N} \\
& \boldsymbol{\xi} \geq \mathbf{0}
\end{array}
$$

where $l$ is a generalized empirical risk function. We will consider only the case where $l$ is a differentiable nondecreasing function satisfying $l(\xi)=0$ and $\frac{\partial l}{\partial \xi}(0)=1$.

Suppose $\mathbf{w}^{*}, b^{*}, \boldsymbol{\xi}^{*}$ is a solution of (17). In the neighbourhood of this solution, defining $\xi=\xi^{*}+\Delta \xi$ (where $\xi^{*}$ is a constant and $\Delta \boldsymbol{\xi}$ is sufficiently small), we may solve (17) by first replacing terms $l\left(\xi_{i}\right)$ with a first-order Taylor approximation of $l$ about $\xi_{i}^{*}$ and then solving the resulting optimisation problem. So, replacing $l\left(\xi_{i}\right)$ terms appropriately, dropping constant terms $l\left(\xi_{i}^{*}\right)$ and adding constant terms $\xi_{i}^{*} \frac{\partial l}{\partial \xi}\left(\xi_{i}^{*}\right)$ we get the simplified training problem:

$$
\begin{array}{ll}
\min L(\mathbf{w}, b, \boldsymbol{\xi})=\frac{1}{2} \mathbf{w}^{T} \mathbf{w}+\frac{C}{N} \sum_{i \in \mathbb{Z}_{N}}\left[\frac{\partial l}{\partial \xi}\left(\xi_{i}^{*}\right)\right] \xi_{i} \\
\text { such that: } \quad d_{i}\left(\mathbf{w}^{T} \boldsymbol{\varphi}\left(\mathbf{x}_{i}\right)+b\right) \geq 1-\xi_{i} \quad \forall i \in \mathbb{Z}_{N} \\
& \boldsymbol{\xi} \geq \mathbf{0}
\end{array}
$$

Now, in our I-FSVM $\infty$ algorithm, if we select:

$$
l(\xi)=\int_{0}^{\xi} h(\zeta) d \zeta
$$

then, upon termination, $s_{i}=h\left(\xi_{i}^{*}\right)=\frac{\partial l}{\partial \xi}\left(\xi_{i}^{*}\right)$, and the solution found at step 2 of our algorithm will actually solve (18) and hence (17) also. From this it may be seen that our algorithm is actually a bilevel [13] method of finding the solution to the generalised primal training problem (17), where the inner level repeatedly solves a linearly approximated version of (17) (ie. (18)) and the outer level fine tunes this approximation by adjusting $\mathbf{s}$ at each step.

For the I-FSVM $\infty$ the generalised empirical risk functions corresponding to the membership functions (15) and (16) are, respectively: 
TABLE I

$$
l_{\text {cnt }}\left(\xi_{i}\right)= \begin{cases}0 & \text { if } \xi_{i} \leq 0 \\ \xi_{i} & \text { if } 0 \leq \xi_{i} \leq 1 \\ 1+\ln \left(\xi_{i}\right) & \text { if } \xi_{i} \geq 1\end{cases}
$$

and:

$$
l_{\text {sig }}\left(\xi_{i}\right)=\tanh \left(\xi_{i}\right)
$$

Now, in the standard SVM we implicitly use the empirical risk function $l(\xi)=\xi$. As $\xi_{i}$ is just the (scaled) distance between the training vector image $\varphi\left(\mathbf{x}_{i}\right)$ and the decision surface in feature space if the vector is misclassified (and 0 otherwise), we see that this particular choice applies a linear distance based error penalty. However, it may be argued that this does not provide a particularly good measure of empirical risk, as in practice all that matters when measuring (and minimising) empirical risk is whether a given training vector is (or is not) correctly classified. Using $h_{\mathrm{cnt}}$ or $h_{\mathrm{sig}}$ the I-FSVM $\infty$ applies an effective empirical risk function that becomes less sensitive to $\xi$ as $\xi$ increases, and so gives a result somewhere between the standard SVM and what may be achieved by considering only the correctness of the classification. Using $h_{\mathrm{sig}}$, in particular, provides a risk function that is in essence a smoothed step penalty.

\section{EXPERIMENTAL RESUlts}

In this section we apply our method to three standard datasets from the UCI repository [8], and compare the results achieved with those achieved using a standard (non-fuzzy) SVM and the fuzzy SVM method of [7].

All code for this experiment was written in $\mathrm{C}++$, and all simulations were performed on a $3.2 \mathrm{GHz}$ Pentium D 940 processor based machine with $4 \mathrm{~GB}$ of RAM running Ubuntu Linux 6.06 (Dapper Drake). The SVM optimizer code was based on a modified version of the SVMHeavy [14] SVM implementation.

\section{A. Methodology}

We have chosen to consider three binary datasets from the UCI repository [8], namely:

1) A subset of the adult (census) database (adult): This dataset contains 1650 training vectors (395 representing wage earnings of more than $\$ 50,000$ per year, the remainder representing wage earnings of less than $\$ 50,000$ per year), with 123 binary attributes per vector. See [15] for details of the data preprocessing methodology used.

2) Spambase database (spambase): This dataset contains 4601 training vectors (1813 spam, remainder nonspam), with 57 attributes per vector (where we have normalised each attribute to zero mean and unit variance). The previous best accuracy reported in [8] for this dataset is $\approx 93 \%$.

3) Abalone dataset (abalone): This dataset contains 4177 training vectors (2096 representing abalone less than 10 years old, the remainder representing abalone at least 10 years old), with 8 attributes per vector.
RESULTS SUMMARY FOR THE ADULT DATASET USING A LINEAR KERNEL, WHERE IN ALL CASES $\frac{C}{N}=30$. INCLUDED IS THE NUMBER OF SUPPORT VECTORS $\left(N_{S}\right)$, THE NUMBER OF ERROR VECTORS $\left(N_{E}\right)$, AND THE 5-FOLD CROSS VALIDATION ACCURACY (5-FOLD). AS WE ARE

USING A LINEAR KERNEL FOR THIS DATASET INPUT SPACE AND FEATURE SPACE ARE THE SAME, AND HENCE FSVM (FEATURE) IS THE SAME AS FSVM (INPUT).

\begin{tabular}{|c||c|c|c|}
\hline & $N_{S}$ & $N_{E}$ & 5 -fold \\
\hline \hline SVM & 446 & 367 & $83.44 \%$ \\
\hline FSVM (input) & 440 & 358 & $83.52 \%$ \\
\hline I-FSVM $1_{\text {cnt }}$ & 446 & 367 & $83.75 \%$ \\
\hline I-FSVM $3_{\text {cnt }}$ & 407 & 325 & $84.77 \%$ \\
\hline I-FSVM $7_{\text {cnt }}$ & 362 & 283 & $85.16 \%$ \\
\hline I-FSVM $\infty_{\text {cnt }}$ & 325 & 243 & $84.30 \%$ \\
\hline I-FSVM $1_{\text {sig }}$ & 446 & 367 & $83.75 \%$ \\
\hline I-FSVM $3_{\text {sig }}$ & 380 & 299 & $85.39 \%$ \\
\hline I-FSVM $\mathbf{7}_{\text {sig }}$ & $\mathbf{3 0 4}$ & $\mathbf{2 2 5}$ & $\mathbf{8 7 . 1 9} \%$ \\
\hline I-FSVM $\infty_{\text {sig }}$ & 298 & 226 & $81.25 \%$ \\
\hline
\end{tabular}

For each of these datasets we considered seven alternative methods of generating fuzzy membership values, namely:

1) SVM: Standard SVM approach (no fuzzification).

2) FSVM (input): Fuzzy SVM with membership function (10) applied in input space.

3) FSVM (feature): Fuzzy SVM with membership function (10) applied in feature space.

4) I-FSVM $n_{\text {cnt }}$ : Iterative fuzzy SVM with membership calculation as per section IV with $h=h_{\mathrm{cnt}}$, with termination after $n$ iterations, where $n \in\{1,3,7\}$.

5) I-FSVM $\infty_{\text {cnt }}$ : Iterative fuzzy SVM with membership calculation as per section IV with $h=h_{\mathrm{cnt}}$ continued until s converges.

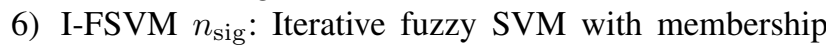
calculation as per section IV with $h=h_{\text {sig }}$, with termination after $n$ iterations, where $n \in\{1,3,7\}$.

7) I-FSVM $\infty_{\text {sig }}$ : Iterative fuzzy SVM with membership calculation as per section IV with $h=h_{\text {sig }}$ continued until s converges.

For all iterative fuzzy methods the learning rate used was $\mu=0.3$, and for FSVM methods $\delta=0.1$. Kernels were selected from linear, polynomial up to $6^{\text {th }}$-order and Gaussian RBF for a range of $\gamma$ values, and $\frac{C}{N}$ was selected from the set $\frac{C}{N} \in\{0.1,0.3,1,3,10,30, \ldots, 100000,300000\}$. For each dataset both the kernel function and the regularisation parameter $\frac{C}{N}$ were selected to achieve optimal 5 -fold cross validation accuracy for the standard SVM. It may be argued that as parameters were selected in this way it will follow that the 5 -fold cross-validation error will not be an ideal measure of generalisation. However they do give a valid measure of the comparative generalisation performance of the different methods, albeit biased in favour of the standard SVM (as the parameters, while optimal for the standard SVM, may not be optimal for the fuzzy variants). 
TABLE II

RESULTS SUMMARY FOR THE SPAMBASE DATASET USING A GAUSSIAN RBF KERNEL WITH $\gamma=100$, WHERE IN ALL CASES $\frac{C}{N}=100$.

INCLUDED IS THE NUMBER OF SUPPORT VECTORS $\left(N_{S}\right)$, THE NUMBER OF ERROR VECTORS $\left(N_{E}\right)$, AND THE 5 -FOLD CROSS VALIDATION ACCURACY (5-FOLD).

\begin{tabular}{|c||c|c|c|}
\hline & $N_{S}$ & $N_{E}$ & 5 -fold \\
\hline \hline SVM & 653 & 238 & $93.45 \%$ \\
\hline FSVM (input) & 655 & 245 & $93.48 \%$ \\
\hline FSVM (feature) & 654 & 262 & $93.51 \%$ \\
\hline I-FSVM $1_{\text {cnt }}$ & 653 & 238 & $90.98 \%$ \\
\hline I-FSVM $3_{\text {cnt }}$ & 632 & 224 & $91.33 \%$ \\
\hline I-FSVM $7_{\text {cnt }}$ & 617 & 212 & $91.71 \%$ \\
\hline I-FSVM $\infty_{\text {cnt }}$ & 592 & 200 & $93.42 \%$ \\
\hline I-FSVM $1_{\text {sig }}$ & 653 & 238 & $90.92 \%$ \\
\hline I-FSVM $3_{\text {sig }}$ & 610 & 209 & $91.49 \%$ \\
\hline I-FSVM $7_{\text {sig }}$ & 557 & 184 & $92.34 \%$ \\
\hline I-FSVM $\infty_{\text {sig }}$ & $\mathbf{4 1 7}$ & $\mathbf{1 6 2}$ & $\mathbf{9 3 . 8 6} \%$ \\
\hline
\end{tabular}

TABLE III

RESULTS SUMMARY FOR THE ABALONE DATASET USING A POLYNOMIAL (CUBIC) KERNEL, WHERE IN ALL CASES $\frac{C}{N}=10$. INCLUDED IS THE NUMBER OF SUPPORT VECTORS $\left(N_{S}\right)$, THE NUMBER OF ERROR VECTORS

$\left(N_{E}\right)$, AND THE 5 -FOLD CROSS VALIDATION ACCURACY (5-FOLD).

\begin{tabular}{|c||c|c|c|}
\hline & $N_{S}$ & $N_{E}$ & 5 -fold \\
\hline \hline SVM & 2080 & 2072 & $78.97 \%$ \\
\hline FSVM (input) & 2030 & 2023 & $78.63 \%$ \\
\hline FSVM (feature) & 2030 & 2023 & $78.63 \%$ \\
\hline I-FSVM $1_{\text {cnt }}$ & 2080 & 2072 & $74.85 \%$ \\
\hline I-FSVM $3_{\text {cnt }}$ & 1918 & 1909 & $76.91 \%$ \\
\hline I-FSVM $7_{\text {cnt }}$ & 1763 & 1755 & $78.75 \%$ \\
\hline I-FSVM $\infty_{\text {cnt }}$ & 1684 & 1676 & $79.13 \%$ \\
\hline I-FSVM $1_{\text {sig }}$ & 2080 & 2072 & $75.90 \%$ \\
\hline I-FSVM $3_{\text {sig }}$ & 1807 & 1800 & $77.82 \%$ \\
\hline I-FSVM $\mathbf{7}_{\text {sig }}$ & $\mathbf{1 4 9 5}$ & $\mathbf{1 4 8 9}$ & $\mathbf{8 2 . 2 5} \%$ \\
\hline I-FSVM $\infty_{\text {sig }}$ & 1344 & 1334 & $70.11 \%$ \\
\hline
\end{tabular}

\section{B. Results}

Results for the adult, spambase and abalone datasets are shown in tables I, II and III. In all cases, the iterative fuzzy SVM was able to outperform both the standard SVM and the standard FSVM, and furthermore the $h_{\text {sig }}$ variant was about to outperform the $h_{\mathrm{cnt}}$ variant.

It is interesting to note that for both the adult and the abalone datasets (which represent the more difficult datasets considered insofar as the classification results achieved for these were consistently lower than those achieved for the spambase dataset) the performance of the I-FSVM $\infty_{\text {sig }}$ was actually worse than the I-FSVM $n_{\text {sig }}$ variant. We are unsure as to why this occurs, but surmise that it may be indicative of overfitting in this case if the algorithm is allowed to continue for too many iterations. The question of terminating the algorithm when optimal generalisation accuracy has been achieved (ie. at the turning point where the generalisation capabilities of the SVM begin to decrease due to overfitting) is an interesting issue which may consider in more detail in future work.

Overall, however, these results indicate that the I-FSVM is able to outperform both the standard SVM and the standard
FSVM using the $h_{\text {sig }}$ membership function provided that the number of iterations is selected appropriately.

\section{CONCLUSION}

We have proposed an iterative fuzzy support vector machine (I-FSVM) method for binary classification. In contrast to standard approaches like [7] which make underlying assumptions about the distribution of training data, our method generates membership values based on their positions relative to the SVM decision function. We have shown that, in its IFSVM $\infty$ form, our algorithm is able to generate results equivalent to those generated using a standard (non-fuzzy) SVM with a modified empirical risk function. We have shown that our I-FSVM method outperforms both standard (non-fuzzy) SVM and the FSVM method of [7] when applied to three real-world datasets (adult, spambase and abalone) taken form the UCI repository.

\section{REFERENCES}

[1] C. Cortes and V. Vapnik, "Support vector networks," Machine Learning, vol. 20, no. 3, pp. 273-297, 1995.

[2] I. Guyon, B. Boser, and V. Vapnik, "Automatic capacity tuning of very large VC-dimension classifiers," in Advances in Neural Information Processing Systems, S. Hanson, J. Cowan, and C. Giles, Eds., vol. 5. Morgan Kaufmann, 1993, pp. 147-155.

[3] S. Chen, A. K. Samington, and L. Hanzo, "Support vector machine multiuser receiver for DS-CDMA signals in multipath channels," IEEE Transactions on Neural Networks, vol. 12, no. 3, pp. 604-611, 2001.

[4] S. I. Hill, P. J. Wolfe, and P. J. W. Rayner, "Nonlinear perceptual audio filtering using support vector machines," in Proceedings of the 11th IEEE Signal Processing Workshop on Statistical Signal Processing, August 2001, pp. 488-491.

[5] J. R. G. L., A. Shilton, M. Parker, and M. Palaniswami, "Prediction of cystine connectivity using SVM," Bioinformation, vol. 1, no. 2, pp. 69-74, 2005.

[6] T. Joachims, "Text categorization with support vector machines: learning with many relevant features," in Proceedings of ECML-98, 10th European Conference on Machine Learning, C. Nédellec and C. Rouveirol, Eds., no. 1398. Chemnitz, DE: Springer Verlag, Heidelberg, DE, 1998, pp. 137-142. [Online]. Available: citeseer.ist.psu.edu/article/joachims98text.html

[7] C.-F. Lin and S.-D. Wang, "Fuzzy support vector machines," IEEE Transactions on Neural Networks, vol. 13, no. 2, March 2002.

[8] C. Blake and C. Merz, "UCI repository of machine learning databases (http://www.ics.uci.edu/ mlearn/MLRepository.html)," University of California, Department of Information and Computer Science., 1998.

[9] J. Mercer, "Functions of positive and negative type, and their connection with the theory of integral equations," Transactions of the Royal Society of London, vol. 209, no. A, 1909.

[10] A. Shilton, "Design and training of support vector machines," Ph.D. dissertation, The University Of Melbourne, 2006. [Online]. Available: http://www.ee.unimelb.edu.au/staff/apsh/publications/

[11] A. Shilton, M. Palaniswami, D. Ralph, and A. C. Tsoi, "Incremental training of support vector machines," IEEE Transactions on Neural Networks, vol. 16, no. 1, pp. 114-131, January 2005.

[12] A. Smola and B. Schölkopf, "A tutorial on support vector regression," Royal Holloway College, University of London, UK, Tech. Rep. NeuroCOLT2 Technical Report Series, NC2-TR-1998-030, October 1998.

[13] S. Dempe, Foundations of Bilevel Programming. Dordrecht: Kluwer Academic Publishers, 2002.

[14] A. Shilton, "SVMHeavy: a support vector machine optimiser," 2001. [Online]. Available: http://www.ee.unimelb.edu.au/staff/apsh/svm/

[15] J. Platt, "Sequential minimal optimization: A fast algorithm for training support vector machines," Microsoft, Redmond, Washington, Tech. Rep. 98-14, April 1998. [Online]. Available: citeseer.nj.nec.com/platt98sequential.html 


\section{University Library}

\section{- M M I E E R VA A gateway to Melbourne's research publications}

Minerva Access is the Institutional Repository of The University of Melbourne

Author/s:

Shilton, A;Lai, DTH

Title:

Iterative fuzzy support vector machine classification

Date:

2007-01-01

Citation:

Shilton, A. \& Lai, D. T. H. (2007). Iterative fuzzy support vector machine classification. 2007 IEEE INTERNATIONAL CONFERENCE ON FUZZY SYSTEMS, VOLS 1-4, pp.1396-1401. IEEE, ELECTRON DEVICES SOC \& RELIABILITY GROUP. https://doi.org/10.1109/ FUZZY.2007.4295570.

Publication Status:

Published

Persistent Link:

http://hdl.handle.net/11343/34669 